\title{
INVESTIGATING THE IMPACT OF ADAPTIVE RISK AWARE RESPONSE SYSTEM WITH DS IMPORTANCE FACTORS IN MANETS
}

\author{
Satya Sravani.B ${ }^{1}$, Prabhakara Rao.B ${ }^{2}$, A.S.Roopa Devi.B ${ }^{3}$ \\ PG Student, ECE Department, JNTU Kakinada, AP, India ${ }^{1}$ \\ Professor, ECE Department, JNTU Kakinada, AP, India ${ }^{2}$ \\ Associate Professor, CSE Department, Pragati Engineering College,Kakinada, AP, India ${ }^{3}$
}

\begin{abstract}
MANET is a self-organized, infrastructure less network that is established to provide wireless communication in improvised environment. Due to the dynamic network topology, the network is more vulnerable to attacks, predominantly the routing attacks. These routing attacks are detected and the risk level is estimated, for which the necessary response actions are implemented by the Intrusion Response System. Of these response actions, the existing binary isolation and naïve fuzzy deteriorate the network performance when implemented. Hence, in this paper, An Adaptive Risk Aware Response System with Dempster Shafer theory that includes a notion of importance factors (ARSDSIF) is analysed and implemented. In this system, the importance factors are being assigned to evidences to attain the attack frequency and node reputation value of nodes in the network. These parameters (attack frequency and node reputation value) determine the risk threshold to instigate reliable time-wise isolation actions in the network. The ARSDSIF improves the overall network performance when compared to basic intrusion response actions. The effectiveness of our approach is analysed with respect to several performance metrics using NS-2 simulator.
\end{abstract}

Keywords: MANET, Dempster Shafer theory, importance factors, risk aware response actions

\section{INTRODUCTION}

Mobile Ad hoc NETwork (MANET) is a set of mobile devices (nodes), which over a shared wireless medium communicate with each other without the presence of a predefined infrastructure or a central authority. The member nodes are themselves responsible for the creation, operation and maintenance of the network. Each node in the MANET is equipped with a wireless transmitter and receiver, with the aid of which it communicates with the other nodes in its wireless vicinity. The nodes which are not in wireless vicinity, communicate with each other hop by hop following a set of rules (routing protocol) for the hopping sequence to be followed. For such networks which were established in improvised environments have the liability to generate security threats. These attacks are due to the compromised nodes in the network. Any compromised nodes under an adversary's control could cause significant damage to the functionality and security of its network since the impact would propagate in performing routing tasks. Most of the routing protocols proposed for MANETs [4] assume that every node in the network is cooperative and not malicious however the behaviour of nodes can be analysed only by ceaseless supervision of intrusion detection system. There exist a variety of methods like cryptography, authentication etc. that are implemented in Manets. These methods when introduced in Manet, does not consider the effects of intrusion response actions on the network performance. Sometimes may result in unexpected network partition,

bringing additional damages to the network.

Hence, we consider an adaptive response technique to detect and mitigate with the routing attacks. The technique is based on an extended Dempster-Shafer mathematical theory with notion of importance factors. It involves risk assessment of both intrusions and response actions in Manet which is a nontrivial task due to its involvements of subjective knowledge, objective evidencing and logical reasoning. Subjective knowledge could be gained from previous experience and objective evidence could be attained from observations while logical reasoning requires a formal foundation. In this paper, we make use of Dempster-Shafer mathematical theory of evidence (D-S theory) [14], that offers an alternative to traditional probability theory for representing uncertainty. It accomplishes the task of risk assessment by labelling the evidences with importance factors and belief functions. This mechanism efficiently improves the network performance by detection and appropriate isolation of malicious nodes in the network.

\section{RELATED WORK}

The primary task of routing protocol is to find the path to ensure that every node acquires a recent map of the network and discover shortest pathway to destinations. Efficient routing protocols have been proposed to handle the dynamic routing traffic in MANET. The major categories of routing protocols are: proactive, reactive and 
hybrid routing protocols. OLSR [12] is the proactive routing protocol selected for performing experiments in this approach after verifying its network performance in the credentials of throughput, end-to-end delay, jitter and overhead. In general, OLSR being a proactive approach has minimal overhead, because messages are broadcasted in the network only through selected MPR nodes. However, if routing attacks occurred in the network like black hole attack, fabrication attack and modification of route replies in the network, there exist worst chances of selecting the malicious nodes itself as the MPR nodes. If that is the case, network experiences serious performance degradation. So, inorder to mitigate such situations, appropriate intrusion detection and response mechanisms have to be adopted to combat with the attacks.

Many research efforts have been made to generate preventive solutions of intrusions [10] for protecting the routing protocols in MANET. However, implementations of these are less hopeful to improve the performance as the response actions may sometimes create involuntary network partition. Numerous intrusion detection systems (IDS) for MANET have been existing and are still the active research area. Due to the nature of MANET, most IDS designed are distributed in nature and have a cooperative architecture. As signature -based and anomaly based IDS models are used for wired network, IDS for MANET use specification-based approaches and statisticsbased approaches. Specification-based approaches, for example DEMEM, supervises the network activities and compare them with known attack features, however they failed to combat with new attacks. On the other side, statistics-based approaches, for example Watchdog and Lipad, compare existing network activities with normal behaviour patterns, which result in higher false positives rate than in the specification-based approaches. Because of the false positives in both MANET IDS models, intrusion alerts generated from these models always accompany with some confidence value, which signifies the possibility of attack occurrence.

Intrusion alerts that are utilised in our paper are node reputation value and attack frequency. Any deviation from the threshold value generated by these attack alerts is noted, intrusion response systems (IRS) will implement isolation of malicious nodes. Response actions like naive fuzzy responses and simple binary isolation will take advantage of IDS alerts but involuntary isolation of nodes may cause unexpected network partition. This brings the concept of cost-sensitive MANET intrusion response approach which considers topology dependency and attack damage. The advantage of our solution is that we integrate evidences from IDS to attain a combined evidence for all the local routing table changes and utilises expert knowledge to estimate risk of both attacks and countermeasures with a mathematical reasoning approach like Dempster Shafer theory. This approach involves differentiating and prioritizing different evidences in terms of security and criticality.

\section{III.EXTENDED DEMPSTER SHAFER THEORY OF EVIDENCE}

D-S theory is a prominent methodology for investigating reliability and security in most of the upgraded information systems and other engineering fields, where accurate calibration is difficult. D-S theory is characterized by the following:

1) It provides a way to represent both subjective and objective evidences with basic probability assignment and belief function.

2) It utilises Dempster's rule of combination (DRC) inorder to combine different evidences that are gathered with reasoning in steps.

3) New Extended Dempster's Rule of Combination with a notion of Importance Factors (IF) treats evidences by differentiating and prioritizing among them based on the attack alerts.

4) This approach considers risk factor caused by both intrusions and response actions.

The adaptiveness of this approach allows to effectively combat with MANET routing attacks.

In this paper, risk-aware response mechanism is implemented with extended Dempster Shafer theory with above stated characteristics. This helps to efficiently combat with attacks in MANET by effective intrusion detection and implementation of adaptive time-wise isolation method. In order to evaluate the efficiency of our mechanism, we perform a series of simulated experiments with a proactive MANET routing protocol, Optimized Link State Routing Protocol (OLSR) using NS-2.34 simulator.

\section{A. Importance Factors}

In D-S theory, propositions are represented as subsets of a given set. When a proposition corresponds to a subset of a frame of discernment, it implies that a particular frame discerns the proposition. First, we introduce a notion of importance factors

1) Definition 1: Importance factor (IF) is a positive real number associated with the importance of evidence. Ifs are derived from historical observations or expert experiences.

2) Definition 2: An evidence E is represented by a 2-tuple $(\mathrm{m}$, IF) where $\mathrm{m}$ describes the basic probability assignment function and IF represents the importance factor.

\section{B. Belief Functions}

If Bel1 and Bel2 are two belief functions over the same frame of discernment [3], with basic probability assignments $\mathrm{m} 1$ and $\mathrm{m} 2$ of those respective evidences and the importance factors of these evidences as IF1 and IF2. Also the function $\mathrm{m}$ defined by our proposed DRCIF is weighted and non associative for multiple evidences. Hence if the sequential information is not available for 
some instances, it is appropriate to make the combined evidence consistent with multiple evidences.

Extended D-S evidence model with importance factors in this approach is as follows: Suppose $\mathrm{E}_{1}=\left(\mathrm{m}_{1}, \mathrm{IF}_{1}\right), \mathrm{E}_{2}=$ $\left(\mathrm{m} 2, \mathrm{IF}_{2}\right)$ are two independent evidences with $\mathrm{m} 1, \mathrm{~m} 2$ as basic probability functions of respective evidences and IF1,IF2 as the importance factors of those evidences. Then, the combined evidence $\mathrm{E}$ of $\mathrm{E} 1$ and $\mathrm{E} 2=\left(\mathrm{m}_{1} \oplus \mathrm{m} 2\right.$, $\left.\left(\mathrm{IF}_{1}+\mathrm{IF}_{2}\right) / 2\right)$, where $\oplus$ is Dempster's rule of combination with importance factors. Our combination algorithm supports the requirement and the complexity of our algorithm is extended up to $\mathrm{n}$ evidences represented by $\mathrm{O}(\mathrm{n})$, where $\mathrm{n}$ is the number of evidences. This indicates that approach of extended Dempster-Shafer theory expects no extra computational cost compared to a naive fuzzybased method.

The algorithm for combination of multiple evidences is constructed as follows:

Algorithm : One evidence

1) $|\mathrm{Ep}|=$ size of ( Ep);

2) While $|\mathrm{Ep}|>1$ do

3) Pick two evidences with the least IF in Ep, named E1 and E2 ;

4) Combine these two evidences, $\mathrm{E}=(\mathrm{m} 1 \oplus \mathrm{m} 2,(1 \mathrm{Fl}+1 \mathrm{~F} 2) / 2) ;$

5) Remove El and E2 from Ep;

6) Add E to Ep;

7) End

The Evidences are collected by the IDS and priorities assigned to each of them by means of importance factors on the basis of attack alerts such as attack frequency and node reputation value of nodes in the network. Risk assessment is done for both attacks and their counter measures. Adaptive decisions are taken on the basis of the node behaviour i.e. attacker or not by comparing with the threshold values namely upper risk tolerance and lower risk tolerance. Finally, Intrusion response system will send an alert to all nodes in the network for updation of changes in the routing table.

\section{IV.RISK AWARE RESPONSE MECHANISM}

In this section, an adaptive risk-aware response mechanism [1] as represented in figure 1, is explained that implements adaptive response actions based on quantitative risk estimation and risk tolerance values. Instead of applying simple binary isolation of malicious nodes, this approach adopts an isolation mechanism in a temporal manner based on the risk value.

\section{A. Response to routing attacks}

In risk aware approach, implementation of two different responses is done to combat with different attacks. They are: Routing table recovery and Node isolation.

Copyright to IJARCCE

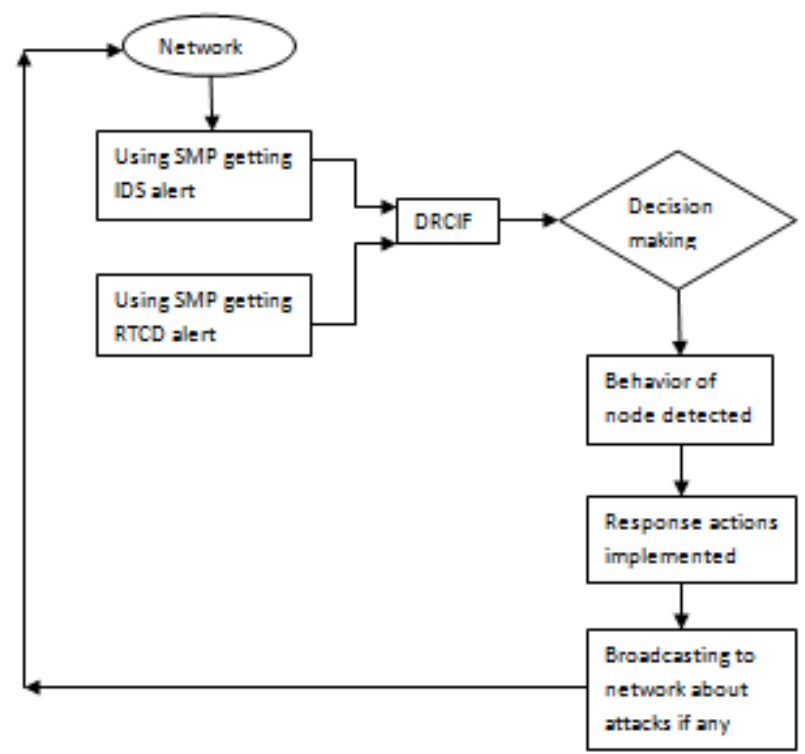

Fig. 1 Risk aware response mechanism

In figure1,SMP represents semi markov process which we use in our Intrusion detection system to attain IDS alert and RTCD alert. Routing table recovery is an appropriate primary response solution after successful detection of attacks by the intrusion detection system. Routing table recovery involves local routing table recovery and global routing recovery. Local routing recovery is done by affected nodes themselves as they detect the attack and automatically updates its own routing table. Global routing recovery involves the process of sending recovered routing messages by victim nodes to all nodes in the network and intimating the available routing table changes in real time for other nodes in MANET. In proactive routing protocols like OLSR, routing table recovery does not bring any additional overhead since it periodically updates the routing table with routing control messages. Hence we also observe that, as long as the detection of attack is positive, the response actions cause no negative impacts on existing routing operations.

Node isolation may be the most intuitive way to eradicate further attacks that may be further imposed by the malicious nodes in MANET. Node isolation response is performed by the neighbours of the malicious node as they ignore the malicious node by neither forwarding packets through it nor accepting any packets from it as soon as they get attack alert. On the other hand, binary node isolation response may yield negative impacts by affecting the routing operations, even bringing more routing damages than the attack itself. In our risk-aware response mechanism, we adopt the following types of time wise isolation responses: no isolation, temporary isolation and permanent isolation.

\section{B. Risk assessment}

Since the response actions after successful detection of intrusions may cause further damages than the attacks itself; hence the risk of both attacks and counter measures should be considered. The security states of MANET are 
under the two categories: (Secure, Insecure). In other words, the frame of discernment [3] would be $\{\varphi$, \{Secure $\}, \quad\{$ Insecure $\}, \quad\{$ Secure, Insecure $\}$. Where Bel $\{$ Insecure $\}$ is used to represent the risk of MANET whereas Bel\{Secure\} is used to represent the secured state of MANET.

1) Evidences Selection: A unified analysis approach for analysing the risks of both attack (RiskA) and countermeasure (Riskc) is implemented. We consider the confidence level of alerts from IDS as the subjective knowledge. For objective knowledge, we consider different routing table modification cases. The routing attack can cause the following changes i.e. existing routing table entry to be missed, or any item of routing table entry to be changed. We illustrate the cases of routing table changes and analyse the degrees of damage from evidences.

2) Combination of evidences: The combined evidence for attacks is $E_{A}$ and the combined evidence for countermeasures is Ec. Thus, Bela (Insecure) and

Belc (Insecure) represent risk of attacks (RiskA) and risk of countermeasures (Riskc), respectively.

Overall Risk $=\operatorname{Bel}_{\mathrm{A}}($ Insecure $)-$ Belc $($ Insecure $)$

\section{Adaptive Decision making}

Adaptive decision making module [6] is dependent on quantitative risk estimation and risk tolerance. The response actions in decision making are divided into multiple bands. Each band is allotted with an isolation degree that presents a different time period for isolation action. The response actions and isolation band boundaries are all decided based on risk tolerance and can be changed when risk tolerance threshold changes. The upper risk tolerance threshold (UT) would signify permanent isolation response whereas the lower risk tolerance threshold $(L T)$ would signify no isolation at all i.e. it maintains each node intact. The band lying between the upper tolerance threshold and lower tolerance threshold is associated with the temporary isolation response, in which the isolation time $(T)$ varies instantaneously. Isolation band, $\mathrm{i}$ is based on the response level where $n$ is the number of bands and $i$ is the corresponding isolation band.

$\mathrm{i}=\frac{\operatorname{Risk}-L T}{U T-L T} \times \mathrm{n}, \operatorname{Risk} \in(L T, U T)$

$\mathrm{T}=100 \times \mathrm{i}$ (milliseconds)

Decision making is based on the risk tolerance threshold and implements the following three levels of isolation.

Risk tolerance thresholds will also be dynamically varied by alerting values, such as attack frequency and node reputation value [9]. If the attack frequency is more, severe response actions i.e. permanent isolation will be implemented to counteract the attack. Implemented riskaware response approach could achieve the isolation of appropriate malicious nodes temporarily and permanently basing on risk factor estimated and narrowing the range between upper and lower risk tolerance thresholds.

1. No isolation: The lower risk tolerance threshold (LT) would result in no isolation and all the nodes in network as such.

2. Temporary isolation: If the risk level falls between the lower risk tolerance thresholds (LT) and higher risk tolerance threshold (HT), the appropriate response action implemented would be temporary isolation.

3. Permanent isolation: If the risk level is above the higher tolerance threshold (HT), the decision would be permanent isolation of node.

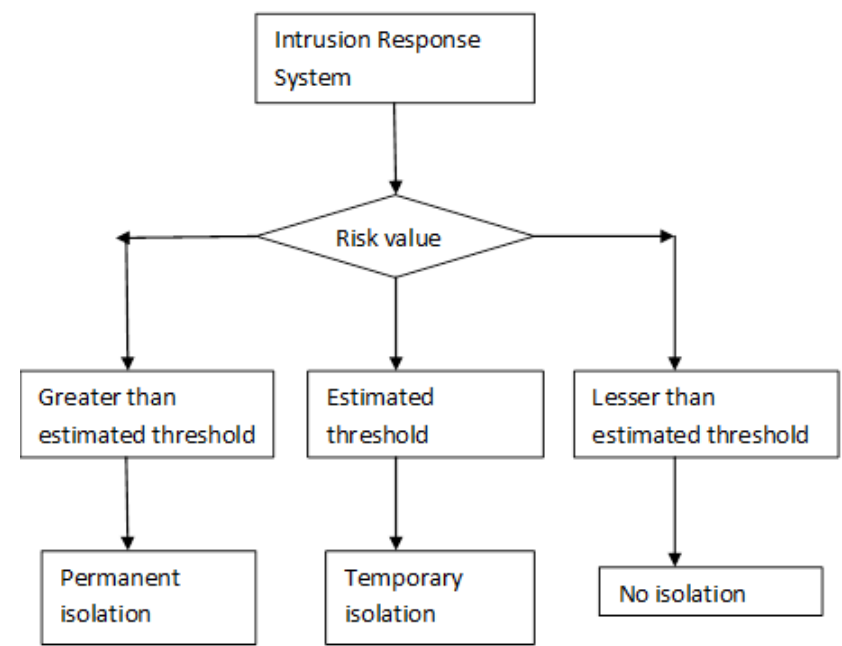

Fig 2. Time wise isolation of nodes based on node reputation and attack frequency

\section{SIMULATION SETUP}

MANET scenarios are generated in NS-2 that are constructed with a topology of 50 nodes with $750 \mathrm{~m} \times 750 \mathrm{~m}$ terrain region. In the conducted experiments, OLSR routing protocol is employed which outperforms other routing protocols in different data traffic models in different performance metrics [15] as it is basically a table driven protocol. The total simulation time was set to 1,200 seconds. Constant Bit Rate (CBR) traffic was used to send 512 byte-UDP packets between nodes. The queuing capacity of every node was set to 150. We adopted a random traffic generator in the simulation that chose random pairs of nodes and sent packets between them. Every node kept track of all packets sent by itself and the entire packet received from other nodes in the network.

\section{A. Performance Evaluation}

In order to evaluate the effectiveness of our adaptive risk aware response solution, we compared the network performance interms of six metrics.

1)Packet delivery ratio: The ratio between the number of packets originated by the application layer CBR sources and the number of packets received by the CBR sink at the final destination. 


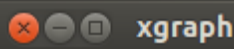

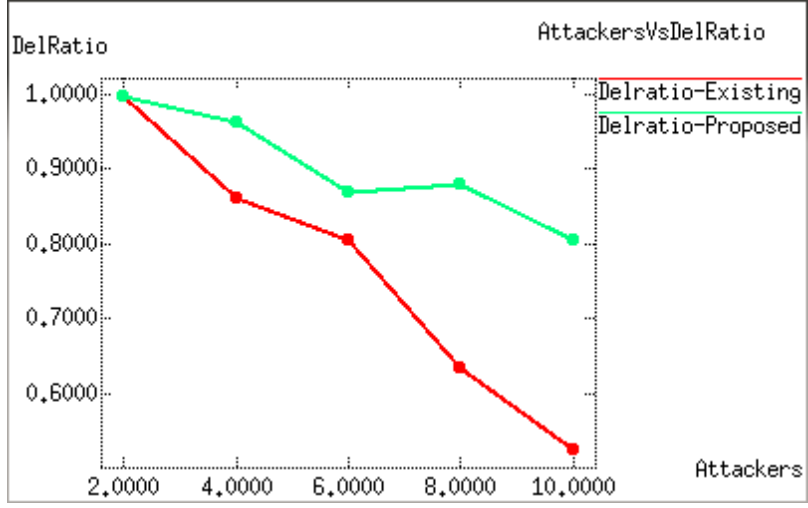

Fig 3. Number of attackers vs. packet delivery ratio

When the number of attacker nodes is rising from $0-6$, the performance of both approaches remained equal, however figure 3 illustrates that after 6 nodes the performance of DRC is degrading drastically and when the DRCIF is implemented, packet delivery ratio is improved to a considerable extent. This is due to the fact that there exists more routing choices for the packets to be delivered in DRCIF scheme.

2) Routing cost: The ratio between the total bytes of routing packets transmitted during the simulation and the total bytes of packets received by the CBR sink at the final destination.

From figure 4, it is clear that the performance of DRCIF is consistently decreased when compared to DRC with varying attacker nodes from 0-10.Routing cost for the proposed DRCIF is reduced since the method is a statistical approach that better analyses and predicts the number of hops included.

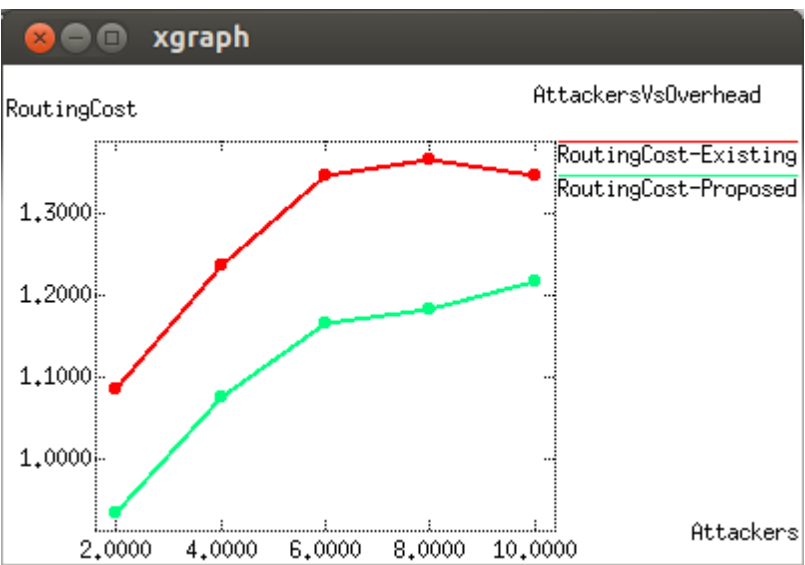

Fig 4. Number of attackers vs. routing cost

3) Control overhead: The number of transmitted routing packets; for example, a HELLO or TC message sent over four hops would be counted as four packets in this metric.

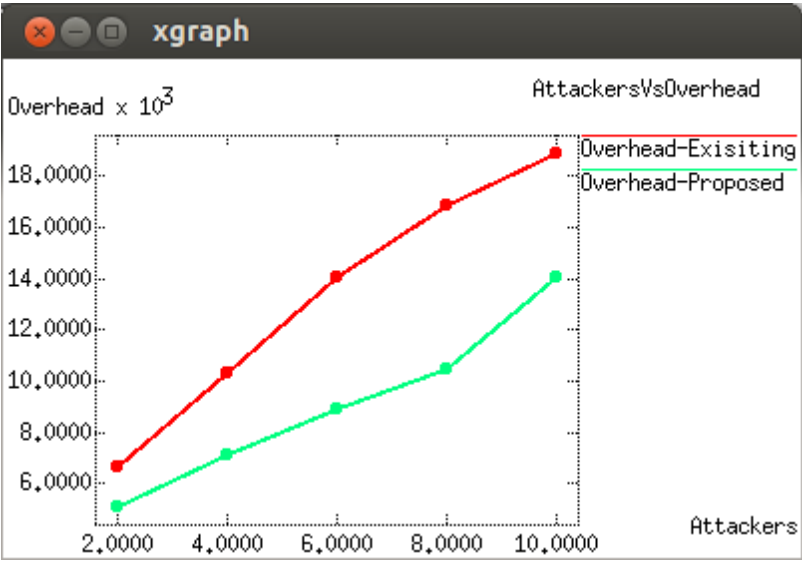

Fig 5.Number of attackers vs. control overhead

From figure 5, it is observed that the overhead of proposed DRCIF scheme is predominantly low when compared to DRC system, due to instigation of appropriate time wise isolation of nodes in the methodology, and the number of nodes permanently isolated is also very low when compared to binary isolation and naïve fuzzy schemes.

4) Mean latency: The average time elapsed from "when a data packet is first sent" to "when it is first received at its destination."

Figure 6 shows that mean latency period is also reduced to noticeable extent in the proposed DRCIF scheme with the variation of attacker nodes from 0 to 10 .

\section{$\otimes \odot$ (ㅁ) $x g r a p h$}

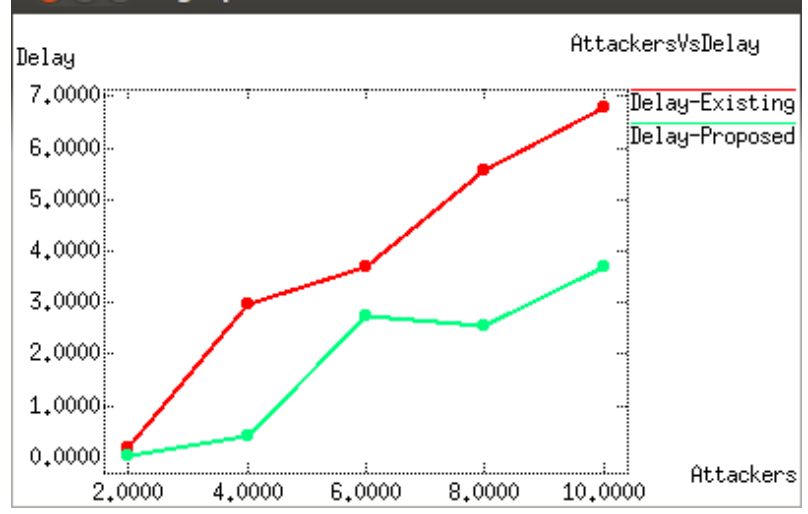

Fig 6. Number of attackers vs. mean latency

5) Energy consumption: The amount of energy consumed from initial state to final state during the transmissions by nodes in the network. 


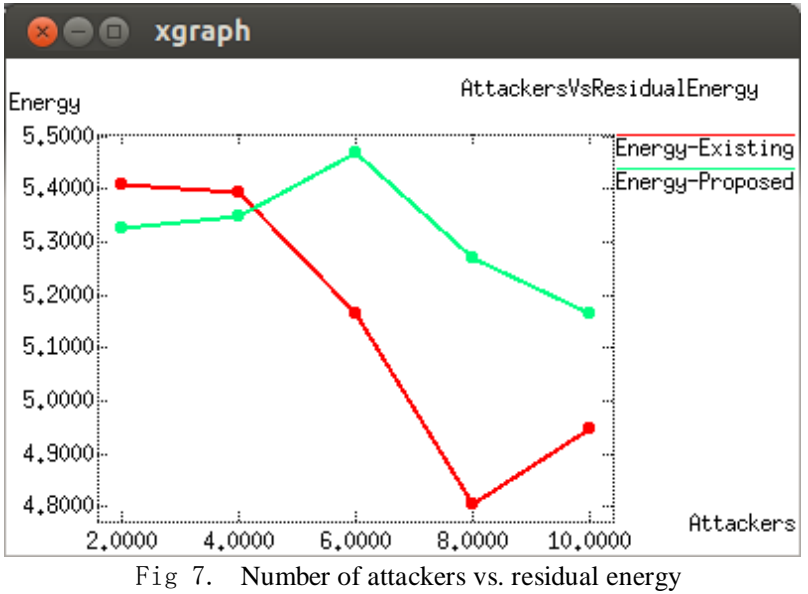

Energy consumption in MANET scenario is high as the nodes are mobile nature. Residual energy in nodes is reduced with the increased number of attacker nodes in the network. However, from figure 7 , we observe that the energy remained in the nodes is comparatively high in the DRCIF scheme when compared to existing DRC scheme, as the impact of attackers is lowered by subsequent detection and implementation of response actions in the network.

\section{CONCLUSIONS}

In this project, risk-aware response solution has been proposed for mitigating MANET routing attacks. The routing attacks in the network have been identified based on the performance variation. This D-S mathematical model has been implemented to identify the possibility of the attack occurrence in the network. Thus, the consideration of the potential damages of attacks and countermeasures have been identified and reduced. In order to measure the risk of both attacks and countermeasures, an extended Dempster- Shafer theory of evidence with a notion of importance factors based on the node reputation value, historical observations and expert experiences. Based on several performance metrics, the network performance has been estimated and compared it with the existing system. We also investigated the performance and practicality of our approach and the experiment results clearly demonstrated the effectiveness and scalability of our risk-aware approach. Based on the performance results obtained through these work, we better analysed the frequency of the attack occurrence in the network.

\section{ACKNOWLEDGEMENT}

I am very thankful to our Prof. Dr. B. Prabhakara Rao and Assoc prof. B.A.S. Roopa Devi for their consistent support and guidance throughout the project period. I acknowledge the support of my peers who supported me in this work.

\section{REFERENCES}

[1]. Ziming Zhao, Hongxin Hu, Gail-Joon Ahn, Ruoyu Wu,"Risk aware mitigation for manet routing attacks", ieee transactions on dependable and secure computing, vol. 9, no. 2, march/april 2012
[2]. Ziming Zhao, Hongxin Hu, Gail-Joon Ahn, and Ruoyu Wu," RiskAware Response for Mitigating MANET Routing Attacks", IEEE Globecom 2010 proceedings.

[3]. Mu, X. Li, H. Huang and S. Tian, "Online Risk Assessment of Intrusion Scenarios Using D-S Evidence Theory", Proc. 13th Europeon Symp. Research in Computer Security (ESORICS '08), pp.35-48, 2008.

[4]. C.S.R.Murthy and B.S.Manoj, Ad Hoc Wireless Networks, Pearson Education, 2008.

[5].O. Basir and X. Yuan. Engine fault diagnosis based on multi-sensor information fusion using Dempster-Shafer evidence theory. Information Fusion, 8(4):379-386, 2007.

[6] P. Cheng, P. Rohatgi, C. Keser, P. Karger, G. Wagner, and A. Reninger. Fuzzy MLS: An Experiment on Quantified RiskAdaptive Access Control, IEEE Symposium on Security and Privacy, volume 2007.

[7]. B. Kannhavong, H. Nakayama, Y. Nemoto, N. Kato and A. Jamalipur, "A Survey of Routing Attacks in Mobile Ad Hoc Networks", IEEE Wireless Comm. Magazine, vol. 14, no. 5, pp.8591, Oct 2007

[8]. Y. Sun, W. Yu, Z. Han, and K. Liu, "Information Theoretic Framework of Trust Modeling and Evaluation for Ad Hoc Networks," IEEE J. Selected Areas in Comm., vol. 24, Feb. 2006

[9]."A Reputation-based Mechanism for Isolating Selfish Nodes in Ad Hoc Networks", M.Tamer Refaei, Vivek Srivastava, Luiz DaSilva,Mohamed Eltoweissy, Networking and Services(MobiQuitous '05) 2005.

[10]. Hao Yang, Haiyun Luo, Fan Ye, Songwu Lu and Lixia Zhanng, "Security on Mobile Ad Hoc Networks: Challenges and Solutions" 1536-1284/04/IEEE Wireless Communica-tions Feb, '04.

[11]. P. Dewan, P. Dasgupta and A. Bhattacharya, "On using Reputations in ad hoc networks to counter malicious nodes",Proceedings of the 10th Intl. Conference on Parallel and Distributed Systems, July 2004, pp. 665-672.

[12]. T. Clausen and P. Jacquet, "Optimized Link State Routing Protocol," Network Working Group, 2003.

[13]. K. Sentz and S. Ferson, "Combination of Evidence in DempsterShafer Theory," technical report, Sandia Nat'1 Laboratories, 2002

[14]. S. Marti, t. Giuli, K. Lai, and M. Baker,"Mitigating Routing Misbehavior in Mobile Ad hoc Networks", Proc. ACM MobiCom, pp. 255-265,2000

[15]. B. Satya Sravani, Dr.B.Prabhakar Rao,B.RoopaDevi, " Estimating the performance differentials of different protocols with varying data traffic", IJAREEIE, vol. 3, issue 7, Jul 2014.

\section{BIOGRAPHIES}

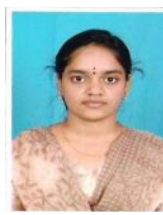

B Satya Sravani has completed her B.E. in Electronics and Communication Engineering from Regency Institute of Technology, Pondicherry University, Puducherry, India in the year 2012. She is currently pursuing her Master's Degree program in Computers and Communication Engineering in J.N.T. University Kakinada, A.P., India..

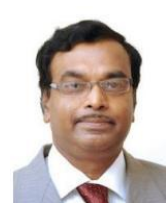

Dr B Prabhakara Rao obtained B.Tech \& M.Tech from S.V. University, Tirupathi with Specializations in Electronics and Communications Engineering, Electronic Instrumentation and Communications Systems in the years 1979 and 1981 respectively. He received the Doctoral degree from Indian Institute of Science, Bangalore in the area of Sonar Signal processing in the year 1995. Currently, he is the senior professor in Electronics and Communication Engineering in J.N.T. University, Kakinada, A.P., India. 
International Journal of Advanced Research in Computer and Communication Engineering Vol. 3, Issue 10, October 2014

B A S Roopa Devi, has completed her B.Tech in Computer Science \& Engineering, J.N.T. University Hyderabad, A.P. India in the year 2004, M.Tech in Software Engineering, J.N.T. University Hyderabad, A.P. India in the year 2006. She is currently working as an associate professor in Pragathi Engineering College, J.N.T University, Kakinada, A.P., India. 